\title{
Kiel Institute for the World Economy
}

\author{
Duesternbrooker Weg 120 \\ 24105 Kiel, Germany
}

Kiel Working Paper No. 1390

\section{Arbeitslosengeld II: \\ Arbeitsanreize und Verteilungsgerechtigkeit}

\author{
von \\ Christos Koulovatianos, Ulrich Schmidt und \\ Carsten Schröder
}

Dezember 2007

The responsibility for the contents of the working papers rests with the author, not the Institute. Since working papers are of a preliminary nature, it may be useful to contact the author of a particular working paper about results or caveats before referring to, or quoting, a paper. Any comments on working papers should be sent directly to the author. 


\title{
Arbeitslosengeld II: Arbeitsanreize und Verteilungsgerechtigkeit*
}

\author{
Christos Koulovatianos \\ Universität Frankfurt, Center for Financial Studies Frankfurt und Universität Wien
}

\section{Ulrich Schmidt ${ }^{\dagger}$}

Universität Kiel und Institut für Weltwirtschaft, Kiel

\section{Carsten Schröder}

Universität Kiel

\section{Zusammenfassung:}

Um die Arbeitsanreize und die Verteilungsgerechtigkeit des Arbeitslosengeldes II (ALG II) zu analysieren, ist eine Quantifizierung des Arbeitsleides und des Wertes der Haushaltsproduktion in verschiedenen Haushaltstypen notwendig. Da diese Größen nicht direkt beobachtbar sind, verwendet die vorliegende Studie Befragungsdaten. Es zeigt sich, dass das ALG II zu zwei Grundproblemen führt: Zum einen sind die Arbeitsanreize gerade für kinderreiche Haushalte sehr gering, zum anderen werden Mehrpersonenhaushalte, insbesondere Familien mit Kindern beim ALG II finanziell benachteiligt. Um diese Probleme zu mildern, sollten Maßnahmen vorangetrieben werden, die den Lebensstandard von Familien mit Kindern im Rahmen des ALG II erhöhen und gleichzeitig die Opportunitätskosten einer Arbeitsaufnahme senken. Geeignet erscheint hier insbesondere eine Ganztagsbetreuung von Kindern mit kostenfreien Mahlzeiten.

\footnotetext{
* Wir danken Alfred Boss, Stefan Homburg, Harmen Lehment, Tim Lohse, Klaus Schrader, Dennis J. Snower, Jürgen Stehn und Michael Stremlau für hilfreiche Kommentare.

${ }^{\dagger}$ Korrespondenz an: Prof. Dr. Ulrich Schmidt, Lehrstuhl für Finanzwissenschaft, Sozialpolitik und Gesundheitsökonomik, Christian-Albrechts-Universität zu Kiel, Olshausenstr. 40, D24098 Kiel, Germany, Telefon: (+49) (431) 880-1400, Telefax: (+49) (431) 880-4621, e-mail: uschmidt@bwl.uni-kiel.de.
} 


\section{Einleitung}

Ziel der sogenannten Hartz-Gesetze war eine Absenkung der Arbeitslosigkeit in Deutschland, was vor allem durch einen gesteigerten Anreiz zur Arbeitssuche und -aufnahme von Arbeitslosen erreicht werden sollte. Ein Kernpunkt dieser Gesetze war die Zusammenlegung von Sozialhilfe und Arbeitslosenhilfe in Form des Arbeitslosengeldes II (ALG II) auf dem Niveau der Sozialhilfe, die im Januar 2005 erfolgte. Die Anreize, die das ALG II bzw. die frühere Sozialhilfe zur Arbeitsaufnahme setzen, wurden bereits in zahlreichen Studien untersucht (siehe bspw. Boss 2002; Boss und Elender 2005a, b; Boss u.a. 2005; Christensen 2004, 2005a, b; Cichorek u.a. 2005a, b; Kohns und Weidmann 2003).

Im Zuge der aktuellen Diskussion um eine „Fortentwicklung“ des ALG II will die vorliegende Studie diese Frage noch einmal aufgreifen und die Arbeitsanreize des ALG II unter Berücksichtigung von Arbeitsleid und Ausfall der Haushaltsproduktion analysieren: Die Aufnahme einer Erwerbstätigkeit verringert das frei verfügbare Zeitbudget im Haushalt, das für Freizeitaktivitäten und Leistungen im Haushaltskontext (z.B. Zubereitung von Mahlzeiten, Kinderbetreuung, etc.) zur Verfügung steht. Diese Faktoren blieben bei den vorherigen Studien - abgesehen von einer indirekten Berücksichtigung in Christensen (2005 a) weitgehend unberücksichtigt, da sie nicht direkt beobachtbar und daher schwer zu messen sind.

Unsere Studie beruht auf einer Arbeit von Koulovatianos u.a. (2007), die Befragungsdaten nutzt, um Informationen über Arbeitsleid und Ausfall der Haushaltsproduktion zu erhalten. Basierend auf diesen Daten kann unsere Studie Antworten auf die folgende Frage liefern: „Wie hoch muss das Arbeitsentgelt sein, damit ein ALG II-Empfänger in Abhängigkeit von seiner Familiensituation bei Annahme eines Arbeitsplatzes den gleichen Lebensstandard erreicht wie bei weiterem Bezug von ALG II und Verzicht auf Arbeitsaufnahme?“ Offensichtlich besteht nur dann ein direkter Anreiz zur Annahme eines Arbeitsplatzes, wenn der Lebensstandard bei Annahme mindestens so hoch ist wie bei Ablehnung. Dabei ist zu berücksichtigen, dass der Nettoverdienst bei Annahme des Arbeitsplatzes unter Umständen deutlich höher als das ALG II sein muss, damit ein Arbeitsanreiz besteht. Dies ist vor allem aus zwei Gründen möglich: Zum einen kann die Arbeitsaufnahme einen direkten Nutzenverlust in Form von Arbeitsleid mit sich bringen, zum anderen besteht aufgrund der geringeren frei verfügbaren Zeit eine eingeschränkte Möglichkeit zur Haushaltsproduktion, was zu Nutzenverlusten bzw. Mehraufwendungen (beispielsweise durch externe 
Kinderbetreuung) führt. Es zeigt sich, dass bei Berücksichtigung dieser Faktoren relativ hohe Löhne notwendig sind, um einen ausreichenden Arbeitsanreiz zu sichern. So wird bei einem Paar mit drei Kindern ein Bruttoarbeitslohn in Höhe von 2567,89 EUR benötigt, damit der gleiche Lebensstandard wie bei Bezug von ALG II entsteht. Dabei sind Zuverdienstmöglichkeiten beim ALG II oder Schwarzarbeit noch nicht einmal berücksichtigt. Eine exakte Berücksichtigung dieser Faktoren gestaltet sich als schwierig, da sie auch mit einem Verlust an Freizeit einhergehen. Es sollte auch erwähnt werden, dass unsere Betrachtungsweise rein statisch ist, d.h. Arbeitsanreize aufgrund einer $\mathrm{zu}$ erwartenden zukünftigen Besserstellung (Aufbau von Humankapital und damit verbundene Einkommenssteigerungen, Erwerb von Rentenansprüchen, etc.) werden nicht berücksichtigt.

Ein weiteres Ziel unserer Studie ist eine Analyse der Verteilungsgerechtigkeit des ALG II ein Aspekt, der bei den bisherigen Untersuchungen weitgehend außer Acht gelassen wurde. Dabei beschränken wir uns auf eine Analyse der horizontalen Verteilungsgerechtigkeit: das ALG II für einen Single ohne Kinder wird in der derzeitigen Höhe als gegeben angenommen und wir stellen uns die Frage, wie hoch das ALG II für andere Haushaltstypen sein müsste, damit sie den gleichen Lebensstandard wie der Single-Haushalt erreichen. Dabei zeigt sich, dass Mehrpersonenhaushalte, insbesondere Familien mit Kindern, beim ALG II deutlich benachteiligt werden, während kinderlose Singles deutlich besser gestellt werden. Dieses Resultat könnte bei der aktuellen Diskussion über Kinderarmut in Deutschland, die beispielsweise in der vor kurzem erschienen Studie des Kinderhilfswerkes dokumentiert wird, Berücksichtigung finden.

Unsere Studie ist wie folgt aufgebaut. Der nächste Abschnitt stellt kurz die verwendeten Daten aus der Arbeit von Koulovatianos u.a. (2007) dar. Im dritten Abschnitt werden unsere Ergebnisse präsentiert. Abschnitt 4 fasst die Ergebnisse zusammen und enthält einige Schlussfolgerungen.

\section{Das Datenmaterial}

Da Arbeitsleid und Haushaltsproduktion nicht direkt beobachtbar und daher schwer messbar sind, benutzen wir Daten aus einer bundesweiten Internet-Umfrage von Koulovatianos et al. (2007), die im Jahr 2005 mit 164 Befragten durchgeführt wurde. Diese Umfrage beruht auf einer Methode von Koulovatianos et al. (2005) zur Ermittlung äquivalenter Einkommen. Ein äquivalentes Einkommen ist als dasjenige Einkommen definiert, das ein bestimmter 
Haushaltstyp benötigt, um den gleichen Lebensstandard wie ein Referenzhaushalt mit einem vorgegebenen Referenzeinkommen zu erreichen. Dabei wird in der Regel ein kinderloser Single als Referenzhaushalt gewählt. Das Referenzeinkommen nahm in der Studie von Koulovatianos et al. (2007) drei Werte an, nämlich 500 EUR, 2000 EUR und 3500 EUR, wobei diese Größen stets als monatliches Nettoeinkommen inklusive aller Sozialtransfers anzusehen sind.

\begin{tabular}{|l|c|l|l|l|l|}
\hline & $\begin{array}{c}\text { 1 Erwachsener, } \\
\text { nicht berufstätig }\end{array}$ & $\begin{array}{c}\text { 1 Erwachsener, } \\
\text { ganztägig } \\
\text { berufstätig }\end{array}$ & $\begin{array}{c}\text { 2 Erwachsene, } \\
\text { beide nicht } \\
\text { berufstätig }\end{array}$ & $\begin{array}{c}\text { 2 Erwachsene, } \\
\text { einer ganztätig } \\
\text { berufstätig der } \\
\text { andere nicht } \\
\text { berufstätig }\end{array}$ & $\begin{array}{c}\text { Erwachsene, } \\
\text { beide } \\
\text { ganztätig } \\
\text { berufstätig }\end{array}$ \\
\hline 0 Kinder & $\mathbf{5 0 0}$ EUR & & & & \\
\hline 1 Kind & & & & & \\
\hline 3 Kinder & & & & & \\
\hline
\end{tabular}

Tabelle 1: Die Fragebogenstruktur

Zentrales Instrument zur Erhebung äquivalenter Einkommen ist Tabelle 1. In den leeren Zellen sollen die Befragten solche Einkommen angeben (äquivalente Einkommen), die den spezifizierten 19 Haushaltstypen den gleichen Lebensstandard garantieren, den der Single mit dem vorgegebenen Einkommen (in Tabelle 1 sind dies 500 EUR) erreicht. Diese Tabelle ist für alle drei Referenzeinkommen einmal auszufüllen. Im Zuge der Befragung wurden noch folgende sozio-ökonomische Charakteristika der Befragten erhoben: Geschlecht, Zusammensetzung des eigenen Haushaltes, Beruf, Arbeitssituation und Nettoeinkommen des eigenen Haushaltes, Ausbildung, Alter und Anzahl der Geschwister. Die Studie von Koulovatianos et al. (2005) sowie eine kürzlich von der forsa Gesellschaft für Sozialforschung und statistische Analyse $\mathrm{mbH}$ durchgeführte repräsentative Untersuchung haben gezeigt, dass die Ermittlung äquivalenter Einkommen mit Hilfe dieser Art von Fragebögen sehr robust und weitestgehend unabhängig von persönlichen Charakteristika der Befragten oder dem Umfragemedium (persönliche Interviews oder computergestützte Internetbefragungen) ist. Dies wird auch durch die sehr geringen Standardfehler (s.u.) bestätigt. Weitere Details zur Umfrage finden sich bei Koulovatianos et al. (2007) sowie in der Tabelle im Anhang, die die Zusammensetzung unseres Samples näher charakterisiert. 
Tabelle 2 zeigt die Mittelwerte der äquivalenten Einkommen sowie deren Standardfehler für alle 19 Haushaltstypen jeweils für alle drei Referenzeinkommen. Dabei bedeutet N „nicht berufstätig“ und B ,ganztägig berufstätig“. Der Eintrag 1603,96 EUR in der vierten Zeile der vorletzten Spalte bedeutet beispielsweise, dass ein Paar bestehend aus einer nicht berufstätigen und einer ganztägig berufstätigen Person mit einem Kind ein monatliches Nettoeinkommen in Höhe von 1603,96 EUR benötigt, um den gleichen Lebensstandard wie ein kinderloser Single mit 500 EUR zu erreichen. Die Daten in Tabelle 2 bilden die Grundlage für die Analyse im nächsten Abschnitt. Die sehr geringen Standardfehler zeigen, dass sich die Angaben der Befragten in der Regel nicht stark unterscheiden.

\begin{tabular}{|c|c|c|c|c|c|c|}
\hline & & \multicolumn{2}{|c|}{ Singles } & \multicolumn{3}{|c|}{ Paare } \\
\hline & & $N$ & $B$ & $N N$ & $B N$ & $B B$ \\
\hline \multirow{4}{*}{500} & $\begin{array}{l}\text { Keine } \\
\text { Kinder }\end{array}$ & 500 & $\begin{array}{l}903.35 \\
(21.66) \\
\end{array}$ & $\begin{array}{l}977.13 \\
(18.63) \\
\end{array}$ & $\begin{array}{c}1314.33 \\
(33.50)\end{array}$ & $\begin{array}{l}1715.85 \\
(53.18)\end{array}$ \\
\hline & $\begin{array}{c}1 \\
\text { Kind }\end{array}$ & $\begin{array}{l}802.13 \\
(8.43)\end{array}$ & $\begin{array}{l}1227.90 \\
(25.65)\end{array}$ & $\begin{array}{c}1266.10 \\
(20.52)\end{array}$ & $\begin{array}{l}1603.96 \\
(36.27)\end{array}$ & $\begin{array}{c}2070.58 \\
(55.63)\end{array}$ \\
\hline & $\begin{array}{c}2 \\
\text { Kinder }\end{array}$ & $\begin{array}{c}1073.41 \\
(16.59)\end{array}$ & $\begin{array}{c}1498.63 \\
(31.90)\end{array}$ & $\begin{array}{c}1522.38 \\
(27.41)\end{array}$ & $\begin{array}{c}1866.16 \\
(4.36)\end{array}$ & $\begin{array}{c}2371.19 \\
(61.40)\end{array}$ \\
\hline & $\begin{array}{c}3 \\
\text { Kinder }\end{array}$ & $\begin{array}{c}1323.05 \\
(26.58)\end{array}$ & $\begin{array}{c}1758.69 \\
(40.93)\end{array}$ & $\begin{array}{c}1747.35 \\
(34.96) \\
\end{array}$ & $\begin{array}{c}2102.74 \\
(52.20)\end{array}$ & $\begin{array}{l}2645.58 \\
(70.85) \\
\end{array}$ \\
\hline \multirow{4}{*}{2000} & $\begin{array}{l}\text { Keine } \\
\text { Kinder }\end{array}$ & 2000 & $\begin{array}{c}2829.57 \\
(67.48) \\
\end{array}$ & $\begin{array}{c}3227.29 \\
(57.10) \\
\end{array}$ & $\begin{array}{c}3856.16 \\
(89.18) \\
\end{array}$ & $\begin{array}{r}4718.60 \\
(147.11) \\
\end{array}$ \\
\hline & $\begin{array}{c}1 \\
\text { Kind }\end{array}$ & $\begin{array}{c}2460.37 \\
(36.42)\end{array}$ & $\begin{array}{c}3308.08 \\
(82.82)\end{array}$ & $\begin{array}{c}3628.81 \\
(78.24) \\
\end{array}$ & $\begin{array}{r}4287.35 \\
(109.57)\end{array}$ & $\begin{array}{l}5249.54 \\
(168.85)\end{array}$ \\
\hline & $\begin{array}{c}2 \\
\text { Kinder }\end{array}$ & $\begin{array}{c}2812.20 \\
(56.29) \\
\end{array}$ & $\begin{array}{c}3700.30 \\
(98.11) \\
\end{array}$ & $\begin{array}{c}3960.06 \\
(91.18) \\
\end{array}$ & $\begin{array}{r}4622.41 \\
(119.55) \\
\end{array}$ & $\begin{array}{l}5660.76 \\
(181.48) \\
\end{array}$ \\
\hline & $\begin{array}{c}3 \\
\text { Kinder } \\
\end{array}$ & $\begin{array}{c}3149.39 \\
(78.51) \\
\end{array}$ & $\begin{array}{l}4066.74 \\
(117.10) \\
\end{array}$ & $\begin{array}{l}4297.53 \\
(116.45) \\
\end{array}$ & $\begin{array}{r}4969.21 \\
(139.19) \\
\end{array}$ & $\begin{array}{r}6092.07 \\
(203.18) \\
\end{array}$ \\
\hline \multirow{4}{*}{3500} & $\begin{array}{l}\text { Keine } \\
\text { Kinder }\end{array}$ & 3500 & $\begin{array}{c}4540.70 \\
(94.02)\end{array}$ & $\begin{array}{c}5277.44 \\
(92.51)\end{array}$ & $\begin{array}{c}6135.82 \\
(139.01)\end{array}$ & $\begin{array}{l}7432.32 \\
(228.72)\end{array}$ \\
\hline & $\begin{array}{c}1 \\
\text { Kind } \\
\end{array}$ & $\begin{array}{c}3980.95 \\
(31.45) \\
\end{array}$ & $\begin{array}{r}5104.73 \\
(105.89) \\
\end{array}$ & $\begin{array}{r}5752.10 \\
(108.92) \\
\end{array}$ & $\begin{array}{r}6695.43 \\
(157.54) \\
\end{array}$ & $\begin{array}{l}8077.59 \\
(246.22) \\
\end{array}$ \\
\hline & $\begin{array}{c}2 \\
\text { Kinder } \\
\end{array}$ & $\begin{array}{r}4410.34 \\
(63.10) \\
\end{array}$ & $\begin{array}{l}5576.07 \\
(124.63) \\
\end{array}$ & $\begin{array}{r}6175.15 \\
(128.12) \\
\end{array}$ & $\begin{array}{r}7141.49 \\
(177.50) \\
\end{array}$ & $\begin{array}{l}8592.84 \\
(265.15) \\
\end{array}$ \\
\hline & $\begin{array}{c}3 \\
\text { Kinder }\end{array}$ & $\begin{array}{c}4815.52 \\
(94.54) \\
\end{array}$ & $\begin{array}{l}6028.02 \\
(148.12) \\
\end{array}$ & $\begin{array}{r}6594.02 \\
(155.18) \\
\end{array}$ & $\begin{array}{r}7582.62 \\
(201.16) \\
\end{array}$ & $\begin{array}{r}9100.91 \\
(285.35) \\
\end{array}$ \\
\hline
\end{tabular}

Tabelle 2: Äquivalente Einkommen

\section{Ergebnisse}

Zunächst wurden die Höhe des ALG II für die einzelnen Haushaltstypen ermittelt, wobei für die Berechnung der Unterkunftskosten der aktuelle Mietspiegel der Stadt Kiel zu Grunde gelegt wurde. Die berechneten Werte finden sich in der zweiten Spalte von Tabelle 3. In der 
ersten Spalte sind die einzelnen Haushaltstypen dargestellt, wobei E für einen Erwachsenen und $\mathrm{K}$ für ein Kind steht. Mit Hilfe von Tabelle 2 wurde nun ermittelt, welches Lohnniveau jeder Haushaltstyp benötigt, damit er bei Arbeitsaufnahme des Haushaltsvorstandes den gleichen Lebensstandard wie mit ALG II erreicht. Um die Werte in Tabelle 2 von den einzelnen Referenzeinkommen auf das jeweilige Niveau des ALG II anzupassen, wurde (unter Berücksichtigung der Inflation im Zeitraum 2005-2007) ein Polynom zweiten Grades geschätzt. Es wird bei den Berechnungen davon ausgegangen, dass im ALG II beziehenden Haushalt keiner der Erwachsenen berufstätig ist, während bei der Arbeitsaufnahme unabhängig davon, ob es sich um ein Paar oder einen Single handelt - immer nur ein Erwachsener im Haushalt ganztägig berufstätig ist.

\begin{tabular}{rcc} 
ALG II & äquiv. Nettoeinkommen & äquiv. Bruttoeinkommen \\
\hline E 699,83 & $1.226,64$ & $1.830,44$ \\
EK 1.000,20 & $1.505,75$ & $1.986,48$ \\
EKK 1.305,75 & $1.810,21$ & $2.265,76$ \\
EKKK 1.587,25 & $2.103,70$ & $2.538,75$ \\
EE 1.104,20 & $1.461,79$ & $1.861,88$ \\
EEK 1.409,75 & $1.768,91$ & $2.094,60$ \\
EEKK 1.691,25 & $2.059,06$ & $2.306,33$ \\
EEKKK 1.969,25 & $2.356,22$ & $2.567,89$ \\
\hline
\end{tabular}

Tabelle 3: Äquivalente Lohnniveaus in Euro pro Monat

Die Ergebnisse unserer Berechnungen finden sich in der dritten und vierten Spalte von Tabelle 3, wobei die dritte Spalte den benötigten Nettolohn und die vierte Spalte den benötigten Bruttolohn angibt, um eine nutzenäquivalente Position zum Bezug von ALG II zu erreichen. Bei der Umrechnung der Netto- in Bruttoeinkommen folgen wir Bönke und Eichfelder (2007), wobei u.a. angenommen wird, dass Mitgliedschaft in der GKV besteht und Paare verheiratet sind. Tabelle 3 zeigt, dass die benötigten Einkommen mit steigender Haushaltsgröße zunehmen. Auffällig ist, dass sich das benötigte Bruttoeinkommen von Paaren und Haushalten mit nur einem Erwachsenen bei gleicher Kinderzahl kaum unterscheiden. Insgesamt sind die benötigten Bruttoeinkommen gerade bei Haushalten mit Kindern so hoch, dass nur bei gut bezahlten Stellen ein Anreiz zur Arbeitsaufnahme besteht. Betrachtet man beispielsweise Alleinerziehende oder Paare mit drei Kindern und legt das Gehalt im öffentlichen Dienst zu Grunde, würde erst ab der Entgeltgruppe TVöD 13 - also ab der dritthöchsten Stufe - ein Anreiz zur Arbeitsaufnahme bestehen. Insbesondere für die von Arbeitslosigkeit besonders betroffene Gruppe der Geringqualifizierten stellt diese aber eine de 
facto kaum erreichbare Besoldungsstufe dar. Dabei ist noch nicht einmal berücksichtigt, dass die ALG II beziehenden Haushalte ihre Situation durch Hinzuverdienstmöglichkeiten noch verbessern können. Aber auch bei Haushalten ohne Kinder kann das ALG II erhebliche negative Anreizwirkungen haben. So können kinderlose Haushalte ihre Situation durch eine Tätigkeit im öffentlichen Dienst erst ab Entgeltstufe TVöD 8 verbessern.

Zur Beurteilung der Verteilungsgerechtigkeit des ALG II wurden die Werte aus Tabelle 2 mit Hilfe eines Polynoms zweiten Grades, das den statistischen Zusammenhang zwischen Referenzeinkommen und äquivalenten Einkommen widerspiegelt, inflationsbereinigt auf ein Referenzeinkommen in Höhe des ALG II für einen kinderlosen Single (699,83 EUR) angepasst. Somit erhalten wir für alle Haushaltstypen genau das Einkommen, das zu dem gleichen Lebensstandard führt, den ein kinderloser Single mit ALG II erreicht. Die errechneten Werte finden sich in der dritten Spalte von Tabelle 4.

\begin{tabular}{rcccc} 
& ALG II & Bedarf & Differenz & $\%$ \\
\hline E & 699,83 & 699,83 & --- & --- \\
EK & $1.000,20$ & $1.086,09$ & $-85,89$ & $-7,91$ \\
EKK & $1.305,75$ & $1.428,68$ & $-122,93$ & $-8,60$ \\
EKKK & $1.587,25$ & $1.744,71$ & $-157,46$ & $-9,03$ \\
EE & $1.104,20$ & $1.335,03$ & $-230,83$ & $-17,29$ \\
EEK & $1.409,75$ & $1.701,62$ & $-291,87$ & $-17,15$ \\
EEKK & $1.691,25$ & $2.024,96$ & $-333,71$ & $-16,48$ \\
EEKKK & $1.969,25$ & $2.311,68$ & $-342,43$ & $-14,81$ \\
\hline
\end{tabular}

Tabelle 4: Verteilungsgerechtigkeit des ALG II

Tabelle 4 zeigt, dass alle Mehrpersonenhalte ein niedrigeres ALG II beziehen, als notwendig wäre, um sie für den mit einem Anstieg der Haushaltsgröße einhergehenden Mehrbedarf zu kompensieren. Somit ist der kinderlose Single im Rahmen des ALG II der am besten gestellte Haushalt, während insbesondere Paare mit Kindern benachteiligt werden und nur einen niedrigeren Lebensstandard erreichen. Insofern erscheint die aktuelle Diskussion über Kinderarmut im Zusammenhang mit ALG II nachvollziehbar.

\section{Schlussbetrachtung}

Ziel unserer Studie war es, das ALG II im Hinblick auf die Kriterien Arbeitsanreize und Verteilungsgerechtigkeit zu analysieren. Da Haushaltsproduktion und Arbeitsleid in diesem 
Zusammenhang wichtige, jedoch nicht direkt beobachtbare Determinanten sind, haben wir Befragungsdaten als empirische Grundlage unserer Analyse verwendet.

Insgesamt zeigen sich zwei zentrale Probleme der aktuellen Ausgestaltung des ALG II. Erstens sind die negativen Arbeitsanreizwirkungen gerade bei Haushalten mit Kindern beträchtlich. Zweitens werden Mehrpersonenhaushalte, insbesondere Paare mit Kindern, im Vergleich zu kinderlosen Singles benachteiligt. Beide Probleme gemeinsam implizieren auf den ersten Blick konträre Politikempfehlungen. Während die negativen Anreizwirkungen eine Verringerung des ALG II für kinderreiche Haushalte nahe legen würden, scheint aus Sicht der Verteilungsgerechtigkeit eher eine Erhöhung für diese Haushalte angezeigt. Selbstverständlich könnte man die aktuelle Situation auch als zweitbestes Optimum ansehen: Obwohl kinderreiche Haushalte eigentlich höhere Zahlungen benötigen würden, bekommen sie diese nicht, um die ohnehin schon schwierige Anreizsituation nicht noch zu verschärfen. Unseres Erachtens ist es aber kaum zu rechtfertigen, Kinderarmut allein aus Anreizgründen in Kauf zu nehmen, wenn auch andere Lösungsmöglichkeiten des Anreizproblems existieren. Da verschiedene empirische Studien eine steigende Kinderarmut in Deutschland belegt haben und daher mit erheblichen gesellschaftlichen Folgekosten bspw. durch zukünftige Integrationsprobleme $\mathrm{zu}$ rechnen ist, sollte man unserer Ansicht versuchen, den Lebensstandard von Familien mit Kindern im Rahmen des ALG II zu erhöhen. Dies könnte durch eine direkte Erhöhung des ALG II für kinderreiche Haushalte erreicht werden. Über effektivere und schärfere Sanktionen bei Ablehnung zumutbarer Arbeitsplätze (ggf. mit ergänzendem ALG II-Anspruch) könnten die einhergehenden Anreizverluste und Zusatzausgaben der öffentlichen Hand teilweise kompensiert werden. Es existieren auch zahlreiche weitergehende Vorschläge (Lohnsubventionen, Kombilöhne, Beschäftigungsgutscheine, etc.), wie man dieser Anreizminderung eventuell effektiver entgegenwirken könnte. Eine Analyse dieser Vorschläge würde jedoch den Rahmen dieser Studie sprengen.

Unter Umständen ist es jedoch vorteilhaft, statt Sanktionen eine allgemeine Senkung des ALG II einzuführen und dann Belohnungen für kooperatives Verhalten zu vergeben. Obwohl beide Vorgehensweisen aus finanzieller Sicht der Betroffenen äquivalent sind, kann sich ihre Wahrnehmung bei diesen durchaus unterscheiden (Bestrafung vs. Belohnung) und so auch unterschiedliche Reaktionen hervorrufen. Auch Arbeitsverpflichtungen bzw. obligatorische Fortbildungen können die Anreizsituation verbessern. Derartige Programme waren in 
Dänemark auch im Zusammenhang mit hohen Arbeitslosengeld- und Sozialhilfeleistungen sehr erfolgreich (siehe Andersen und Svarer, 2007). Selbstverständlich muss man bei einem Vergleich mit Dänemark die dortige Flexibilität des Arbeitsmarktes berücksichtigen. Gleichzeitig besteht die Hoffnung, dass sich flexiblere Arbeitsmarktreglungen auch in Deutschland politisch leichter durchsetzten lassen, wenn parallel die soziale Sicherung erhöht wird.

Neben den negativen Anreizwirkungen kann gegen eine direkte Erhöhung des ALG II für Familien mit Kindern auch angeführt werden, dass das zusätzliche Geld in vielen Fällen nur zu einer Erhöhung des Konsums von Erwachsenengütern führt, ohne die Situation der Kinder spürbar zu verbessern. In diesem Falle wären Sachleistungen zusätzlichen Geldleistungen vorzuziehen. Eine Maßnahme die in diesem Zusammenhang diskutiert werden sollte, sind ganztägige Kinderbetreuungsangebote (Ganztagsschulen bzw. -kindergärten), die Mahlzeiten und Freizeitgestaltung umfassen und für sozial schwache Familien kostenfrei sind. Derartige Angebote sind aus unserer Sicht zu bevorzugen, da sie gleich mehrere Vorteile besitzen: Zum einen wird durch kostenfreie Mahlzeiten und Freizeitangebote für Kinder die Benachteiligung von kinderreichen Familien im Rahmen des ALG II gemindert. Zweitens würden diese Angebote wohl in jedem Fall zu einer direkten Verbesserung der Situation der Kinder führen. Drittens wird aber auch gleichzeitig die Anreizsituation verbessert: Eine Arbeitsaufnahme verursacht geringere Nutzeneinbußen, da das Zeitbudget der Eltern aufgrund der externen Kinderbetreuung weniger angespannt ist. Insgesamt erscheint somit eine Ausweitung der Kinderbetreuungsangebote selbst bei Vernachlässigung der zu erwartenden positiven Effekte auf die Geburtenrate allein aus Anreizgesichtspunkten als sehr vorteilhaft. 
A Anhang

\begin{tabular}{|c|c|c|}
\hline & \multicolumn{2}{|c|}{$\mathrm{N}=164$} \\
\hline & $\mathbf{N}$ & $\%$ \\
\hline \multicolumn{3}{|l|}{ Geschlecht des Befragten } \\
\hline Weiblich & 56 & 34,1 \\
\hline Männlich & 108 & 65,9 \\
\hline \multicolumn{3}{|l|}{ Partner im Haushalt } \\
\hline $\mathrm{Ja}$ & 69 & 42,1 \\
\hline Nein & 95 & 57,9 \\
\hline \multicolumn{3}{|l|}{ Anzahl der Kinder im Haushalt } \\
\hline 0 & 140 & 85,4 \\
\hline 1 & 18 & 11,0 \\
\hline 2 & 5 & 3,0 \\
\hline 3 oder mehr & 1 & 0,6 \\
\hline \multicolumn{3}{|l|}{ Anzahl der Geschwister des Befragten } \\
\hline 0 & 30 & 18,3 \\
\hline 1 & 51 & 31,1 \\
\hline 2 & 57 & 34,8 \\
\hline 3 oder mehr & 26 & 15,8 \\
\hline \multicolumn{3}{|l|}{ Alter des Befragten } \\
\hline Jünger als 20 Jahre & 0 & 0,0 \\
\hline 20 bis 40 Jahre & 147 & 89,6 \\
\hline Älter als 40 Jahre & 17 & 10,4 \\
\hline \multicolumn{3}{|l|}{ Höchster Bildungsabschluss des Befragten } \\
\hline Kein Schulabschluss & 1 & 0,6 \\
\hline Hauptschule & 1 & 0,6 \\
\hline Realschule & 3 & 1,8 \\
\hline Fachhochschulreife & 2 & 1,2 \\
\hline Abitur & 98 & 59,8 \\
\hline Universitäts- oder Fachhochschulabschluss & 59 & 36,0 \\
\hline \multicolumn{3}{|l|}{ Erwerbsstatus des Befragten } \\
\hline Sozialhilfeempfänger oder Arbeitsloser & 1 & 0,6 \\
\hline Arbeiter & 1 & 0,6 \\
\hline Angestellter & 45 & 27,4 \\
\hline Beamter & 8 & 4,8 \\
\hline Schüler, Auszubildender, Student & 102 & 62,4 \\
\hline Selbständiger & 5 & 3,0 \\
\hline Rentner oder Pensionär & 1 & 0,6 \\
\hline Hausfrau oder -mann & 1 & 0,6 \\
\hline \multicolumn{3}{|l|}{ Arbeitszeit des Befragten } \\
\hline Nicht erwerbstätig & 27 & 16,5 \\
\hline Unregelmäßige, seltene Erwerbstätigkeit & 63 & 38,4 \\
\hline Halbtags erwerbstätig & 25 & 15,2 \\
\hline Ganztags erwerbstätig & 49 & 29,9 \\
\hline \multicolumn{3}{|l|}{ Arbeitszeit des Partners } \\
\hline Nicht erwerbstätig & 117 & 71,3 \\
\hline Unregelmäßige, seltene Erwerbstätigkeit & 6 & 3,7 \\
\hline Halbtags erwerbstätig & 12 & 7,3 \\
\hline Ganztags erwerbstätig & 29 & 17,7 \\
\hline \multicolumn{3}{|l|}{ Verfügbares Haushaltseinkommen pro Monat } \\
\hline Unter EUR 875 & 64 & 39,0 \\
\hline Zwischen EUR 875 und EUR 1625 & 46 & 28,0 \\
\hline Zwischen EUR 1625 und EUR 2375 & 24 & 14,6 \\
\hline Zwischen EUR 2375 und EUR 3125 & 18 & 11,0 \\
\hline EUR 3125 und mehr & 12 & 7,4 \\
\hline
\end{tabular}

Tabelle A1: Zusammensetzung der Stichprobe 


\section{Literaturverzeichnis}

Andersen, T.M., und M. Svarer (2007), Flexicurity - Labour market performance in Denmark. Herauskommend in: CESifo Studies.

Bönke, T., und S. Eichfelder (2007), Verteilungswirkungen von Steuern, Sozialabgaben und Arbeitslosengeld II. Arbeitspapier, Freie Universität Berlin.

Boss, A. (2002), Sozialhilfe, Lohnabstand und Leistungsanreize. Kieler Studien 318, Berlin.

Boss, A., B. Christensen und K. Schrader (2005), Anreizprobleme bei Hartz IV: Lieber ALG II statt Arbeit? Kieler Diskussionsbeiträge, 421, Institut für Weltwirtschaft

Boss, A., und T. Elendner (2005a), Verstärkte Arbeitsanreize durch das Arbeitslosengeld II. Die Weltwirtschaft 2, 168-196.

Boss, A., und T. Elendner (2005b), Incentives to Work: The Case of Germany. Kieler Arbeitspapiere 1237, Institut für Weltwirtschaft, Kiel.

Christensen, B. (2004), Der Einfluss der Agenda 2010 auf die Arbeitslosigkeitsdauer. Zeitschrift für Wirtschaftspolitik 53, 95-119.

Christensen, B. (2005a), Die Lohnansprüche deutscher Arbeitsloser. Determinanten und Auswirkungen von Reservationslöhnen. Kieler Studien 333, Berlin.

Christensen, B. (2005b), Reservationslöhne und Arbeitslosigkeitsdauer. Jahrbücher für Nationalökonomie und Statistik 225, 303-324.

Cichorek, A., S. Koch und U. Walwei (2005a), Arbeitslosengeld II. Erschweren „Zusatzjobs“ die Aufnahme einer regulären Beschäftigung? IAB Kurzbericht (8), Nürnberg.

Cichorek, A., S. Koch und U. Walwei (2005b), Arbeitslosengeld II. Höhere Arbeitsanreize Geplant. IAB Kurzbericht (7), Nürnberg.

Kohns, S., und J. Weidmann (2003), Sozialhilfe reformieren - Arbeitsanreize stärken. Zeitschrift für Wirtschaftspolitik 52, 187-202.

Koulovatianos, C., C. Schröder und U. Schmidt (2005), On the Income Dependence of Equivalence Scales. Journal of Public Economics 89, 967-996.

Koulovatianos, C., C. Schröder und U. Schmidt (2007), Non-Market Household Time and the Cost of Children. Journal of Business and Economic Statistics, forthcoming. 
